\title{
Concentração da vinhaça por microfiltração seguida de nanofiltração com membranas
}

\author{
Maria Thereza de Moraes Santos Gomes ${ }^{(1)}$, Kaliana Sitonio Eça ${ }^{(1)}$ e Luiz Antonio Viotto(1)
}

\begin{abstract}
(1)Universidade Estadual de Campinas, Departamento de Engenharia de Alimentos, Laboratório de Medidas Físicas, Cidade Universitária Zeferino Vaz, Rua Monteiro Lobato, no 80, CEP 13083-862 Campinas, SP. E-mail: mt_msgomes@yahoo.com.br, kali_ufpb@hotmail.com, viotto@fea.unicamp.br
\end{abstract}

Resumo - O objetivo deste trabalho foi avaliar a concentração da vinhaça por meio da microfiltração (MF) seguida de nanofiltração (NF). Os experimentos foram conduzidos em laboratório, a $60^{\circ} \mathrm{C}$ e $500 \mathrm{rpm}$, com uso de vinhaça com $2,8 \%$ de sólidos totais (ST), para avaliação da influência da pressão no fluxo de permeado e na retenção de ST. A pressão variou de 1,0 a 3,4 bar, para MF, e de 15 a 35 bar, para NF. Utilizou-se membrana de celulose, com massa molecular de corte de $100 \mathrm{kDa}$, para MF, e membrana de poliestersulfona (PES), de 300,500 e $1.000 \mathrm{Da}$, para NF. A MF realizada a 1,8 bar foi selecionada, por ter apresentado bom fluxo e menor consumo de energia, e o permeado obtido foi submetido à NF. A NF apresentou retenções de ST de 47-75\%, e o fluxo mais elevado foi obtido com a PES $1.000 \mathrm{Da}$, a 35 bar. O concentrado final apresenta 4\% de ST e é considerado adequado para uso em solo, em termos de demanda química de oxigênio e de teores de ST.

Termos para indexação: fluxo de permeado, membrana de celulose, membrana de poliestersulfona, retenção de sólidos totais.

\section{Vinasse concentration by microfiltration followed by nanofiltration with membrane}

\begin{abstract}
The objective of this work was to evaluate vinasse concentration by microfiltration (MF) followed by nanofiltration (NF). The experiments were carried out in the laboratory, at $60^{\circ} \mathrm{C}$ and $500 \mathrm{rpm}$, using vinasse with $2.8 \%$ total solids (TS) in order to assess the influence of pressure on permeate flux and TS retention. Pressure ranged from 1.0 to 3.4 bar for MF, and from 15 to 35 for NF. A membrane cellulose with molecular weight cutoff of $100 \mathrm{kDA}$ was used for MF, and a polyethersulfone (PES) membrane of 300, 500, and 1,000 Da was used for NF. MF at 1.8 bar was selected, because of its good flux and lower energy consumption, and the permeate obtained was subjected to NF. NF showed TS retention of $47-75 \%$, and the highest permeate flux was obtained with PES $1,000 \mathrm{Da}$ at 35 bar. The final concentrate has $4 \%$ of TS and is considered appropriate for soil use, in terms of chemical oxygen demand and TS content.
\end{abstract}

Index terms: permeate flux, cellulose membrane, polyethersulfone membrane, total solids retention.

\section{Introdução}

A vinhaça, resíduo líquido resultante da etapa de destilação do álcool, é o principal efluente das destilarias, por apresentar alto poder poluidor, considerável volume de produção e elevada potencialidade de uso. Embora haja a presença de matéria orgânica e teores consideráveis de elementos minerais em sua composição química, a vinhaça é constituída principalmente (cerca de 97\%) de água (Silva et al., 1983). De acordo com Freire \& Cortez (2000), a vinhaça apresenta elevadas taxas de demanda bioquímica (42.000-100.000 $\left.\mathrm{mg} \mathrm{L}^{-1}\right)$ e de demanda química de oxigênio (10.000-210.000 $\left.\mathrm{mg} \mathrm{L}^{-1}\right)$. Portanto, sua eliminação de maneira adequada e a baixo custo é fundamental para minimizar os impactos ambientais da produção de álcool.
A aplicação da vinhaça ao solo, como fertirrigação, é uma prática bastante difundida entre as usinas e as destilarias brasileiras, tendo apresentado resultados positivos na produtividade agrícola (Lyra et al., 2003; Silva et al., 2007), com economia na aquisição de fertilizantes (Santana, 1985). Entretanto, a adição de doses elevadas de vinhaça ao solo pode ocasionar um enriquecimento exagerado em sais, o que pode acarretar lixiviação de íons para águas subterrâneas (Glória \& Orlando Filho, 1984).

A Norma Técnica P4.231 (Companhia de Tecnologia de Saneamento Ambiental, 2006) estabelece os critérios e os procedimentos para a aplicação da vinhaça ao solo. Tendo em vista que há elevado conteúdo de água na vinhaça, sua aplicação em áreas mais distantes da destiliaria sofre as limitações resultantes dos mais altos custos de transporte. Assim, a vinhaça concentrada, além 
de adequar-se à norma técnica de distribuição, representa uma possibilidade de viabilizar seu uso em áreas afastadas e descontínuas.

Alguns métodos são descritos na literatura para o tratamento e a concentração da vinhaça. Hulett (1980) propõe um processo de evaporação, enquanto Nicolaiewsky (1981) recomenda um tratamento físico-químico da vinhaça, que consiste de floculação, sedimentação e filtração. Navarro et al. (2000) avaliaram a possibilidade de bioconcentração da vinhaça, ao utilizá-la no preparo do meio de fermentação para produção de etanol. Já Nataraj et al. (2006), relataram o uso de um processo híbrido de nanofiltração e osmose inversa para remoção de cor e contaminantes da vinhaça. Zayas et al. (2007) utilizaram a coagulação/floculação e a oxidação eletroquímica para a purificação de vinhaça biologicamente tratada.

Diversas patentes têm sido registradas para concentração da vinhaça, com emprego de diferentes tipos de evaporadores, como, por exemplo, a patente $\mathrm{n}^{\mathrm{o}}$ 0055239-A1, depositada nos Estados Unidos em 2010, que se refere a um evaporador de múltiplo efeito (Almeida $\&$ Sampaio, 2010). Como o uso de evaporadores apresenta demanda elevada de energia, a avaliação de processos alternativos para a concentração da vinhaça, com menor demanda energética, como o emprego de membranas, apresenta alta relevância. $O$ processo de separação por membranas têm sido bastante utilizado no tratamento de diversos efluentes industriais (Benítez et al., 2009; Acero et al., 2010). Contudo, ainda são necessários estudos sobre o uso da microfiltração seguida de nanofiltração para a concentração da vinhaça.

O objetivo deste trabalho foi avaliar a concentração da vinhaça por meio da microfiltração seguida de nanofiltração.

\section{Material e Métodos}

Utilizou-se um lote de $30 \mathrm{~L}$ de vinhaça, proveniente de Cosmópolis, SP, doado pela Usina Açucareira Ester S.A., recolhido na safra de 2009, em novembro. A vinhaça foi homogeneizada, envasada em sacos de $1 \mathrm{~L}$ e armazenada a $-18^{\circ} \mathrm{C}$, para posterior utilização. Na Tabela 1 , são apresentadas as características físico-químicas da vinhaça utilizada nos experimentos.

Foram utilizadas membranas planas poliméricas da marca comercial Microdyn-Nadir (Microdyn-Nadir, Wiesbaden, Alemanha). Para microfiltração (MF), utilizou-se membrana de celulose (CEL), com massa molecular de corte de $100 \mathrm{kDa}$, que, embora seja tipicamente utilizada na ultrafiltração (UF), é considerada como de MF pelo fornecedor. Para nanofiltração (NF), foram utilizadas membranas de poliestersulfona (PES), de 300, 500 e 1.000 Da.

Os experimentos foram conduzidos em célula de filtração de bancada, com fluxo perpendicular, à temperatura de $60^{\circ} \mathrm{C}$ e agitação magnética de $500 \mathrm{rpm}$. A célula encamisada de aço inox apresentava $260 \mathrm{~mm}$ de altura, $128 \mathrm{~mm}$ de diâmetro externo e $110 \mathrm{~mm}$ de diâmetro interno, tendo sido acoplada a um cilindro de nitrogênio para fornecimento da pressão transmembrana $\left(\mathrm{P}_{\mathrm{TM}}\right)$.

Antes do início de cada processo, as membranas novas foram cortadas em diâmetro de $8,1 \mathrm{~cm}$ e imersas em água destilada, por 30 min, para remoção dos produtos utilizados na conservação da membrana. Em seguida, foi feita permeação com água destilada nas mesmas condições da corrida realizada com a vinhaça, para que a estrutura mecânica das membranas se adaptasse à pressão de operação do sistema.

As amostras da vinhaça foram descongeladas e aquecidas em banho-maria, até $60^{\circ} \mathrm{C}$. Os experimentos foram realizados em três etapas. Na primeira, as corridas de MF foram realizadas em batelada, tendo-se utilizado $800 \mathrm{~mL}$ de vinhaça, nas seguintes pressões: 1,0, 1,8, 2,6 e 3,4 bar, até que um fator de concentração (FC) igual a 4 fosse atingido. Na segunda etapa, foram realizadas repetições nas mesmas condições do processo de MF selecionado na primeira etapa, para acúmulo de permeado, pois houve limitação da capacidade de alimentação $(800 \mathrm{~mL})$ da unidade laboratorial. Na terceira etapa, um volume de $400 \mathrm{~mL}$ do permeado resultante das corridas de acúmulo foi submetido à NF, a 15, 25 e 35 bar, até FC igual a 2. No total, foram realizadas 19 corridas: quatro de MF, para seleção do melhor processo, com observação

Tabela 1. Caracterização físico-química da vinhaça ${ }^{(1)}$.

\begin{tabular}{|c|c|}
\hline Parâmetro & Vinhaça \\
\hline $\mathrm{pH}$ & $3,97 \pm 0,04$ \\
\hline Umidade (\%) & $97,19 \pm 0,07$ \\
\hline Sólidos totais $(\%)$ & $2,81 \pm 0,07$ \\
\hline Sólidos suspensos $\left(\mathrm{mg} \mathrm{L}^{-1}\right)$ & $1.407 \pm 138$ \\
\hline Sólidos dissolvidos $\left(\mathrm{mg} \mathrm{L}^{-1}\right)$ & $25.822 \pm 157$ \\
\hline Cinzas $(\%)$ & $0,79 \pm 0,03$ \\
\hline Potássio (mg L-1) & $1.638 \pm 205$ \\
\hline Sódio $\left(\mathrm{mg} \mathrm{L}^{-1}\right)$ & $812 \pm 99$ \\
\hline Demanda química de oxigênio $\left(\mathrm{mg} \mathrm{L}^{-1}\right)$ & $26.625 \pm 177$ \\
\hline
\end{tabular}

${ }^{(1)}$ Médias \pm desvio-padrão obtidas em triplicata. 
do fluxo de permeado e da retenção de sólidos totais (ST); seis para obtenção do volume de permeado necessário, com uso do processo de MF selecionado anteriormente; e nove corridas de NF.

Foi avaliado o desempenho das membranas de MF e de NF, mediante a quantificação da percentagem de retenção $(\% \mathrm{R})$ de $\mathrm{ST}$ e do fluxo de permeado $(\mathrm{J})$, calculados de acordo com as equações: $\% \mathrm{R}=\left(1-\mathrm{C}_{\mathrm{p}} / \mathrm{C}_{\mathrm{c}}\right)$ $x 100$ e $J=m_{p} / A_{p} x t$, em que: $C_{p}$ e $C_{c}$ são a concentração no permeado e no concentrado, respectivamente; $\mathrm{m}_{\mathrm{p}}$ é a massa de permeado no tempo t; e $\mathrm{A}_{\mathrm{p}}$ é a área de permeação.

A alimentação, o permeado e o concentrado da MF e da NF foram caracterizados quanto aos valores de $\mathrm{pH}, \mathrm{ST}$, sólidos dissolvidos ( $\mathrm{SD}$ ), sólidos suspensos (SS), cinzas, potássio, sódio e demanda química de oxigênio (DQO), tendo-se empregado os procedimentos recomendados pelo Standard Methods for the Examination of Water and Wastewater (2005).

\section{Resultados e Discussão}

$\mathrm{Na} \mathrm{MF}$, não houve permeação de SS pela membrana, ou seja, ocorreu $100 \%$ de retenção, com formação de concentrado com valores médios de $5.515 \mathrm{mg} \mathrm{L}^{-1}$ de SS (Figura 1). As retenções de SD variaram de 24 a $37 \%$, e o aumento da pressão permitiu a obtenção de

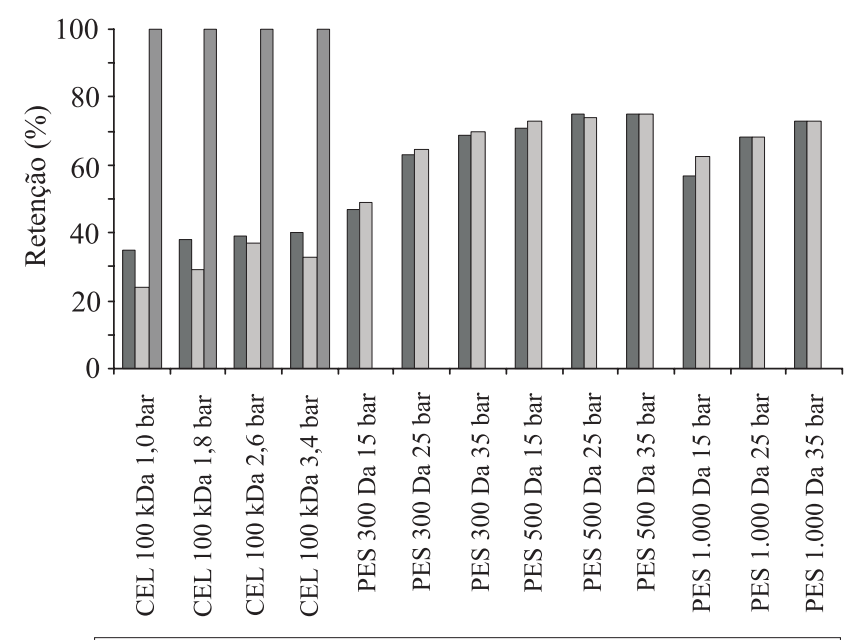

$\square$ Sólidos totais $\square$ Sólidos dissolvidos $\square$ Sólidos suspensos

Figura 1. Percentagem de retenção de sólidos totais (ST), dissolvidos (SD) e suspensos (SS), em função das pressões e das membranas utilizadas nos processos de microfiltração (membranas de celulose-CEL) e nanofiltração (membranas de poliestersulfona - PES) da vinhaça. maior teor de SD no concentrado. Segundo Navarro et al. (2000), os principais SD presentes na vinhaça são os carboidratos não fermentáveis, os sais e alguns compostos, como furfural, glicerol, propanol e ácido lático. $\mathrm{Na} \mathrm{MF}$, a maior retenção de $\mathrm{ST}(40 \%)$ foi observada a 3,4 bar, seguida de $38 \%$ de retenção nas pressões intermediárias e de $35 \%$ a 1,0 bar.

$\mathrm{Na}$ NF, o concentrado apresentou teor de ST entre 3,3 e $4 \%$, e o permeado variou de 1 a $1,7 \%$. Os coeficientes de retenção de ST variaram de 47 a $75 \%$, e as maiores retenções foram observadas na membrana PES $500 \mathrm{Da}$ (71-75\%) e PES $1.000 \mathrm{Da}(57-73 \%)$ (Figura 1). Estas membranas apresentaram uma concentração maior do que as membranas com menor massa molecular de corte, como a de $300 \mathrm{Da}$ (47-69\%), em virtude da camada polarizada adjacente à sua superfície, que atua como uma segunda membrana e aumenta a retenção de sólidos (Obermeyer et al., 1993). A NF apresentou o mesmo comportamento da MF, com maior teor de SD no concentrado à medida que aumenta a pressão.

Nataraj et al. (2006) descreveram um processo híbrido de NF e osmose inversa (OI) para remoção de cor e contaminantes da vinhaça. Em ambos os processos, foram empregados membranas poliamídicas, tipo TFC (composto pelicular fino), em espiral. De acordo com os autores, a NF foi eficaz na remoção de cor e partículas coloidais, na redução de SD totais $(80 \%)$, na condutividade elétrica $(95 \%)$ e na concentração de íons de cloreto (45\%), tendo-se empregado pressão variável de 30-50 bar. O processo sequencial de OI, a 50 bar, resultou em 99\% de redução de DQO, potássio e resíduos de SD totais.

Os valores retidos de cinzas, na MF, variaram de 0,81 a $0,88 \%$, para o concentrado, e de 0,72 a $0,76 \%$, para o permeado (Figura 2), o que indica que as cinzas atravessaram a membrana, em razão de sua baixa massa molecular. $\mathrm{O}$ valor ligeiramente maior no concentrado pode ser explicado pela camada polarizada formada, que foi suficiente para reter parte desses componentes, ou pela interação dos compostos minerais com a própria membrana ou com os outros compostos de maior massa molecular que ficaram retidos.

A vinhaça é fonte de potássio e traz benefícios para o solo (Arienzo et al., 2009), quando aplicada em concentrações que não excedem os limites impostos pela norma técnica P4.231 (Companhia de Tecnologia e Saneamento Ambiental, 2006), segundo a qual a aplicação de vinhaça só pode ser realizada se o teor de potássio disponível no solo for menor do que 5\% da 
capacidade de troca de cátions. A retenção de potássio na MF variou de 1,50 a 13,8\%, com maior retenção na pressão de 3,4 bar (Figura 2). Na NF, as retenções de potássio variaram de 24 a $60 \%$, e as de sódio de 23 a $67 \%$, com as maiores retenções na pressão de 35 bar.

Como os valores de retenção foram bastante próximos (Figura 1), a escolha da membrana foi feita com base nos valores de fluxo de permeado (Figura 3). Os fluxos de permeado apresentaram uma curva típica, descrita em três estágios, conforme

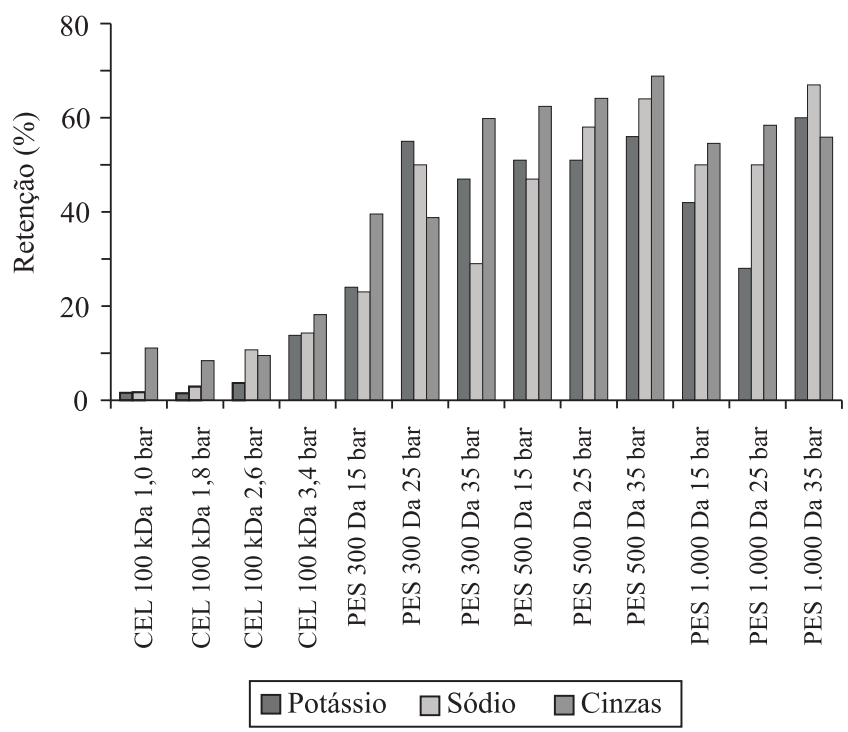

Figura 2. Percentagem de retenção de potássio, sódio e cinzas, em função das pressões e das membranas utilizadas nos processos de microfiltração (membranas de celulose - CEL) e nanofiltração (membranas de poliestersulfona PES), da vinhaça.

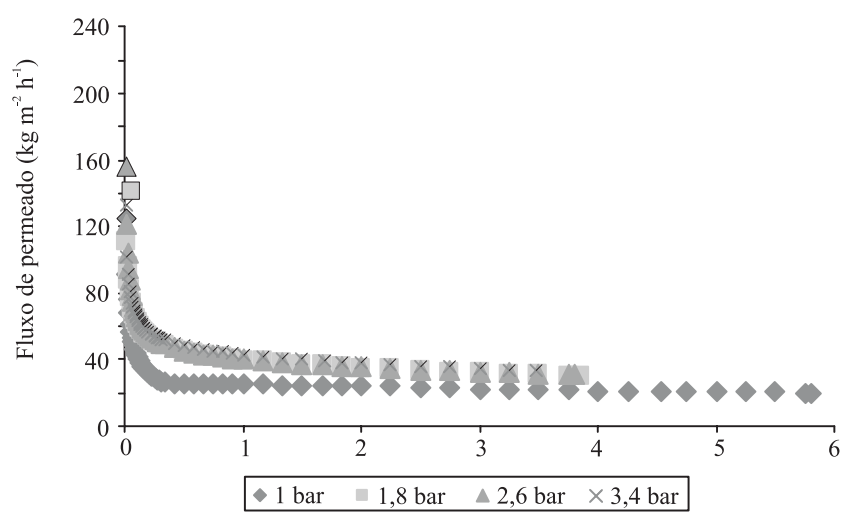

Figura 3. Fluxo de permeado na microfiltração da vinhaça, em diferentes pressões, até fator de concentração igual a 4 .
Marshall \& Daufin (1995): queda brusca do fluxo nos primeiros minutos, em decorrência da polarização ocasionada pela concentração; variação do fluxo decrescente e precipitação dos solutos acumulados, com consequente bloqueio dos poros e adsorção dos componentes na membrana, o que resulta na formação da camada polarizada e na incrustação; e consolidação da incrustação, com redução contínua e lenta no fluxo.

Houve um aumento significativo no fluxo de permeado na mudança de pressão de 1,0 para 1,8 bar (Figura 3). Pressões acima de 1,8 bar tiveram pouca influência nos fluxos, tendo-se observado fluxo a $\mathrm{FC}=4$, de $30,3,30,7$ e $33,4 \mathrm{~kg} \mathrm{~m}^{-2} \mathrm{~h}^{-1}$, para as pressões de $1,8,2,6$ e 3,4 bar, respectivamente. Apesar de o fluxo ter sido levemente superior a 3,4 bar, a pressão de 1,8 bar foi selecionada por ter apresentado bom fluxo e menor consumo de energia.

O comportamento das curvas durante as corridas de acúmulo de permeado na MF alcançou boa reprodutibilidade, o que pode ser confirmado pelos resultados semelhantes obtidos para as seis corridas realizadas nas mesmas condições operacionais (Figura 4).

Durante a NF, a pressão teve grande influência nos fluxos de permeado (Figura 5). O fluxo aumentou nos primeiros minutos e, em seguida, apresentou tendência de se manter estável ou em leve declínio, em virtude da consolidação da incrustação. É provável que o aumento no fluxo de permeado nos primeiros $5 \mathrm{~min}$ da corrida esteja associado à dinâmica da formação da camada polarizada no início do experimento.

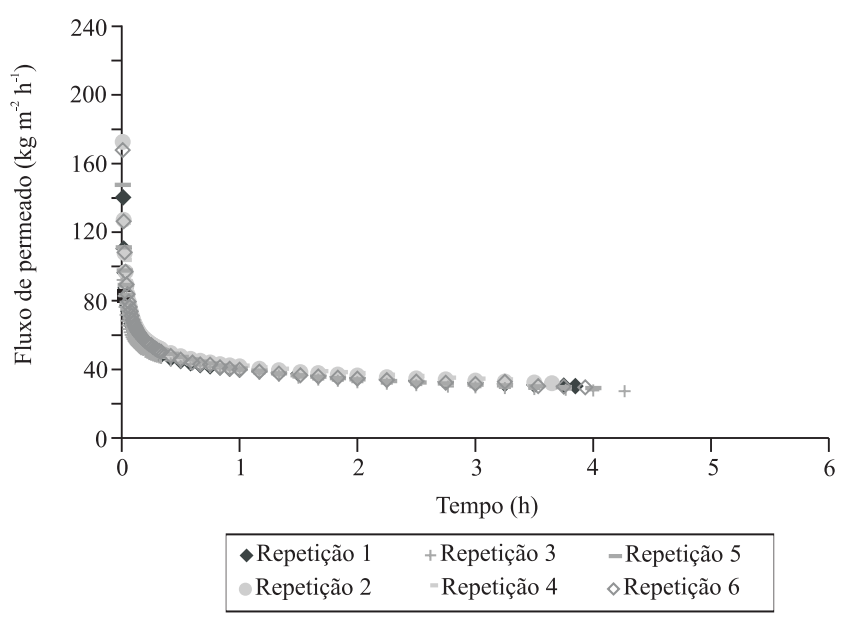

Figura 4. Repetições das corridas de microfiltração da vinhaça, para acúmulo de permeado. 
Os valores de fluxos iniciais e finais variaram para cada membrana, sob as diferentes pressões avaliadas. A membrana PES 1.000 Da apresentou os melhores fluxos (Figura 5), independentemente da pressão. No geral, as membranas PES 300 e $500 \mathrm{Da}$ apresentaram curvas de permeado semelhantes nas pressões avaliadas, e, em alguns casos, a PES $300 \mathrm{Da}$ apresentou maior fluxo que a $500 \mathrm{Da}$, como na pressão de 25 bar. Possivelmente, a maior abertura na membrana de 500 Da permitiu a passagem de impurezas que levaram ao entupimento e ao menor fluxo de permeado neste caso.

As melhores condições do processo combinado de MF e NF foram obtidas com uso da membrana CEL $100 \mathrm{kDa}$, a 1,8 bar, e da membrana PES 1.000 Da, a 35 bar. A alimentação da $\mathrm{MF}$ a $\mathrm{FC}=4$, com membrana de CEL $100 \mathrm{kDa}$, apresentou 2,9\% de ST, tendo formado permeado com $2,5 \%$ e concentrado com $4,0 \%$ de $\mathrm{ST}$. Este permeado seguiu para $\mathrm{NF}$ a $\mathrm{FC}=2$, tendo formado permeado e concentrado com 1,0\% e $3,9 \%$ de ST, respectivamente. O permeado resultante da NF apresenta-se estéril e pode ser utilizado em outros processos, tais como retorno à fermentação e embebição da moenda. Além disso, sua concentração por OI também pode ser uma opção viável. No processo de NF, o concentrado apresentou DQO cerca de três vezes superior ao permeado (Tabela 2). Já na MF, a DQO no concentrado quase dobrou em relação ao permeado. Entretanto, Ludovice (1997) observou que a DQO da vinhaça diminui ao longo do perfil do solo. De fato, Lyra et al. (2003), ao avaliar o potencial poluidor da vinhaça sobre a qualidade de água do

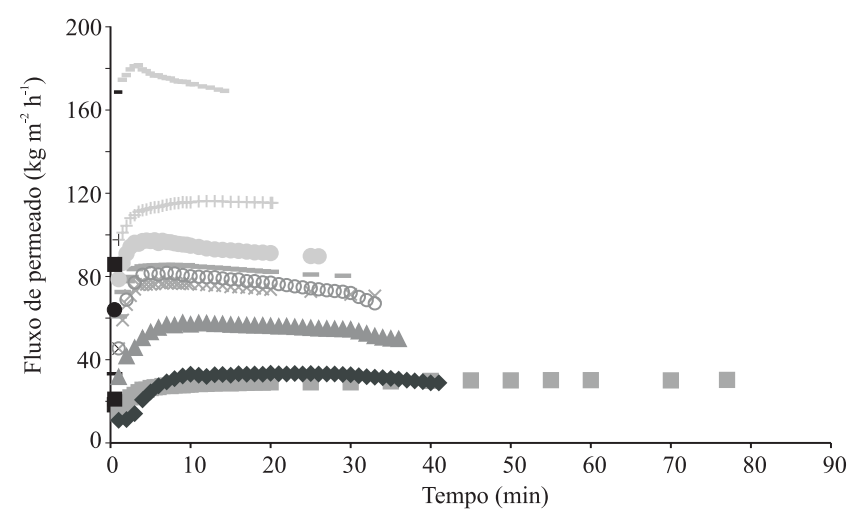

15 bar $300 \mathrm{Da} \times 25$ bar $300 \mathrm{Da}-35$ bar $300 \mathrm{Da} \bullet 15$ bar $500 \mathrm{Da} \Delta 25$ bar $500 \mathrm{Da}$ ○35 bar $500 \mathrm{Da} 15$ bar $1.000 \mathrm{Da} 25$ bar $1.000 \mathrm{Da}-35$ bar $1.000 \mathrm{Da}$

Figura 5. Fluxo de permeado na nanofiltração da vinhaça até fator de concentração igual a 2 .
Tabela 2. Demanda química de oxigênio (DQO) na alimentação (A), no permeado (P) e no concentrado (C) de vinhaça submetida aos tratamentos de microfiltração e nanofiltração.

\begin{tabular}{lcccc}
\hline Processo & Membrana & Pressão & Fonte & DQO (mg L $\left.{ }^{-1}\right)$ \\
\hline \multirow{2}{*}{ Microfiltração } & CEL 100 kDa & \multirow{2}{*}{1,8 bar } & P & $26.750 \pm 1.090$ \\
& & & C & $39.417 \pm 1.010$ \\
\hline \multirow{3}{*}{ Nanofiltração } & PES 1.000 Da & \multirow{2}{*}{35 bar } & P & $10.583 \pm 520$ \\
& & & C & $35.167 \pm 577$ \\
\hline
\end{tabular}

lençol freático, concluíram que o solo é eficiente na redução da DQO. Brito \& Rolim (2005), ao utilizar vinhaça in natura com DQO de $48.860 \mathrm{mg} \mathrm{L}^{-1}$, em experimento de incubação, também obtiveram ótimas taxas de remoção no lixiviado, independentemente da dose aplicada. Assim, apesar de o concentrado ter apresentado, em média, valor de DQO 54\% superior à alimentação, o poder de remoção da matéria orgânica contida neste resíduo, pelo solo, é capaz de viabilizar o uso de vinhaça concentrada.

\section{Conclusões}

1. A concentração da vinhaça por microfiltração seguida de nanofiltração é possível.

2. O concentrado final obtido é adequado para o uso em solo, em termos de demanda química de oxigênio e de teores de sólidos totais.

\section{Agradecimentos}

À Coordenadoria de Aperfeiçoamento de Pessoal de Nível Superior, pelo apoio financeiro; e à usina Ester S.A., pela doação da vinhaça.

\section{Referências}

ACERO, J.L.; BENETÍZ, F.J.; LEAL, A.I.; REAL, F.J.; TEVA, F. Membrane filtration technologies applied to municipal secondary effluents for potential reuse. Journal of Hazardous Materials, v.177, p.390-398, 2010.

ALMEIDA, J.L. de; SAMPAIO, P.H. Evaporator of vinasse concentration for distilleries in general. US patent 0055239-A1, 4 Mar. 2010.

ARIENZO, M.; CHRISTEN, E.W.; QUAYLE, W.; KUMAR, A. A review of the fate of potassium in the soil-plant system after land application of wastewaters. Journal of Hazardous Materials, v.164, p.415-422, 2009. 
BENÍTEZ, F.J.; ACERO, J.L.; LEAL, A.I.; GONZÁLEZ, M. The use of ultrafiltration and nanofiltration membranes for the purification of cork processing wastewater. Journal of Hazardous Materials, v.162, p.1438-1445, 2009.

BRITO, F.L. de; ROLIM, M.M. Comportamento do efluente e do solo fertirrigado com vinhaça. Agropecuária Técnica, v.26, p.60-67, 2005.

COMPANHIA DE TECNOLOGIA DE SANEAMENTO AMBIENTAL. P4.231: vinhaça: critérios e procedimentos para aplicação no solo agrícola. São Paulo: CETESB, 2006. 12p.

FREIRE, W.J.; CORTEZ, L.A.B. Vinhaça de cana-de-açúcar. Guaíra: Agropecuária, 2000. 203p.

GLÓRIA, N.; ORLANDO FILHO, J. Aplicação de vinhaça: um resumo e discussões sobre o que foi pesquisado. Álcool e Açúcar, v.16, p.32-39, 1984.

HULETT, D. Concentração do vinhoto. Sugar y Azúcar do Brasil, v.1, p.35-36, 1980.

LUDOVICE, M.T.F. Estudo do efeito poluente da vinhaça infiltrada em canal condutor de terra sobre o lençol freático. 1997. 117p. Dissertação (Mestrado) - Universidade Estadual de Campinas, Campinas.

LYRA, M.R.C.C.; ROLIM, M.M.; SILVA, J.A.A. da. Toposseqüência de solos fertigados com vinhaça: contribuição para a qualidade das águas do lençol freático. Revista Brasileira de Engenharia Agrícola e Ambiental, v.7, p.525-532, 2003.

MARSHALL, A.D.; DAUFIN, G. Physico-chemical aspects of membrane fouling by dairy fluids. In: FOULING and Cleaning in Pressure Driven Membrane Processes. Brussels: International Dairy Federation, 1995. p.8-35.

NATARAJ, S.K.; HOSAMANI, K.M.; AMINABHAVI, T.M. Distillery wastewater treatment by the membrane-based nanofiltration and reverse osmosis processes. Water Research, v.40, p.2349-2356, 2006.

NAVARRO, A.R.; SEPÚlVEDA, M. Del C.; RUBIO, M.C. Bio-concentration of vinasse from the alcoholic fermentation of sugar cane molasses. Waste Management, v.20, p.581-585, 2000.

NICOLAIEWSKY, E. Tratamento físico-químico do vinhoto: floculação, sedimentação e filtração. Brasil Açucareiro, v.98, p.23-30, 1981.

OBERMEYER, H.D.; KULOZIK, U.; KESSLER, H.G. Controlled deposit formation to influence the retention of solutes in reverse osmosis and ultrafiltration. Desalination, v.90, p.161-172, 1993.

SANTANA, S.S. Economicidade da aplicação de vinhaça em comparação à adubação mineral. Álcool e Açúcar, v.23, p.26-38, 1985.

SILVA, L.C.F.; ZAMBELLO JUNIOR, E.; ORLANDO FILHO, J.; ARRUDA FILHO, H.C. Utilização da vinhaça seca e das cinzas de caldeira como fertilizantes na cultura de cana-de-açúcar. STAB, v.1, p.35-41, 1983.

SILVA, M.A.S. da; GRIEBELER, N.P.; BORGES, L.C. Uso de vinhaça e impactos nas propriedades do solo e lençol freático. Revista Brasileira de Engenharia Agrícola e Ambiental, v.11, p.108-114, 2007.

STANDARD methods for the examination of water and wastewater. $21^{\text {st }}$ ed. Washington: American Public Health Association, 2005. Irregular paging.

ZAYAS, T.; RÓMERO, V.; SALGADO, L.; MERAZ, M.; MORALES, U. Applicability of coagulation/flocculation and electrochemical processes to the purification of biologically treated vinasse effluent. Separation and Purification Technology, v.57, p.270-276, 2007.

Recebido em 30 de setembro de 2010 e aprovado em 4 de maio de 2011

Pesq. agropec. bras., Brasília, v.46, n.6, p.633-638, jun. 2011 